\title{
SPECTRAL METHODS FOR A GENERALIZED PROBABILITY THEORY
}

\author{
BY \\ STANLEY P. GUDDER( $\left.{ }^{1}\right)$
}

1. Introduction. A generalized probability theory arises naturally from an attempt to formulate a statistical model for quantum mechanics. One soon finds that the probability theory of Kolmogorov is inadequate for the description of a quantum mechanical system mainly because the quantum mechanical events fail to form a $\sigma$-algebra of subsets of a set. Instead, they have a much less richly endowed algebraic structure which we shall a "logic". We therefore look upon generalized probability as a stochastic theory in which the events form a logic. Besides being useful in the study of quantum mechanics, this theory has a purely mathematical interest since it constructs a stochastic formalism on an algebraic structure and at the same time gives an abstract generalization of Hilbert space theory.

The first formulation of this theory is due to G. Bodiou [1], [2]. More recently, V.S. Varadarajan [8] has emphasized the stochastic properties of an axiomatic formulation of quantum mechanics due to G. W. Mackey [5] and has proved some important results in this theory. The author heartily recommends these references for the background and motivation of this study. In this paper, the main interest centers on the spectral theory of the subject. The spectrum is important physically since it represents the "allowable" values of a quantum mechanical observable. It is also mathematically useful since it introduces topological methods into the formalism.

2. Definitions and notation. Let $L$ be a complemented lattice. That is, $L$ is a lattice with first and last elements, 0,1 respectively and $a \rightarrow a^{\prime}$ satisfying the conditions

(i) $\left(a^{\prime}\right)^{\prime}=a$ for all $a \in L$;

(ii) $a \leqq b$ implies $b^{\prime} \leqq a^{\prime}$;

(iii) $a \vee a^{\prime}=1$ for all $a \in L$.

Received by the editors July 20, 1964, and, in revised form, November 5, 1964 .

( ${ }^{1}$ ) This paper is based on a dissertation submitted to the University of Illinois at which time the author was partially supported by the National Science Foundation. The manuscript was revised and completed at the Mathematics Research Center,United States Army, Madison Wisconsin, under Contract No.:DA-11-022-ORD-2059. The author would like to thank Professor Robert G. Bartle for his help and encouragment. 
We say that $a, b \in L$ are disjoint and write $a \perp b$ if $a \leqq b^{\prime}$. If $a \perp b$ we shall use the notation $a+b$ for $a \vee b$. A complemented lattice satisfying the following two conditions is called an orthocomplemented lattice or $\operatorname{logic}\left({ }^{2}\right)$.

(iv) $a \leqq b$ implies there exists $c \in L$ such that $a+c=b$;

(v) if $\left(a_{i}\right)_{1}^{\infty}$, are mutually disjoint, then $\sum a_{i} \in L$.

It is shown in [8] that if $a \leqq b$ there exists a unique $c$ such that $a+c=b$ and in fact $c=a^{\prime} \wedge b$. If $a+c=b$ we write $c=b-a$. By a state on $L$ we mean a non-negative function $m$ on $L$ satisfying

(1) $m(1)=1$;

(2) $m\left(\sum a_{i}\right)=\sum m\left(a_{i}\right)$.

A logic is called full in case:

(3) if $a, b \in L$ and $a \neq b$, there exists a state $m$ such that $m(a) \neq m(b)$;

(4) for every $a \neq 0$ there exists a state $m$ such that $m(a)=1$.

We will henceforth let $L$ denote a full logic with elements $a, b, c, \cdots$ and $M$ its set of states. Note that $L$ becomes a Boolean $\sigma$-algebra if the distributive law, $a \wedge(b \vee c)=(a \wedge b) \vee(a \wedge c)$ holds for every $a, b, c \in L$.

LEMMA 2.1. If L is a Boolean $\sigma$-algebra then axioms (3) and (4) are equivalent.

Proof. We first show (3) implies (4). Suppose $a \in L$ and $a \neq 0$. Then there exists $m \in M$ such that $m(a) \neq 0$. Define $m_{1}$ by $m_{1}(b)=m(a \wedge b) / m(a)$ for every $b \in L$. Now $m_{1} \in M$ and $m_{1}(a)=1$. To show that (4) implies (3), suppose $a \neq b$. If either $a$ or $b$ is 0 then (3) follows directly. Therefore, we suppose that $a, b \neq 0$. Either $a-a \wedge b \neq 0$ or $b-a \wedge b \neq 0$, so suppose, for definiteness, the former holds. Therefore, there exists $m \in M$ such that $m(a-a \wedge b)=1$. Now $a=(a-a \wedge b)+(a \wedge b)$ and $b=(b-a \wedge b)+(a \wedge b)$ and we have $m(a)=1$ $m(b)=0$.

Definitions. Let $A$ be a Boolean $\sigma$-algebra. A $\sigma$-homomorphism $u \rightarrow x(u)$ of $A$ into $L$ is a mapping which satisfies:

(i) $x(0)=0, x(1)=1$;

(ii) $u \perp v$ implies $x(u) \perp x(v)$;

(iii) $x\left(\sum u_{i}\right)=\Sigma x\left(u_{i}\right)$.

Denote the complex plane by $C$ and the real line by $R$. A $\sigma$-homomorphism from the Borel subsets $B(C)$ of $C$ into $L$ is called a derivable. If $x$ is a derivable satisfying $x(E)=0$ for all $E \in B(C)$ such that $E \cap R=\varnothing$, then $x$ is called an observable.

Notice that there is essentially no difference between an observable and a $\sigma$ homomorphism based on $B(R)$. For this reason we shall subsequently not distinguish between the two concepts.

${ }^{2}$ ) Note that our definition of a logic differs from [8] in that we assume a logic to be a lat tice while the latter does not. However Proposition 3.11 of [8] is false as it stands (a counterexample in [6] was communicated to the author by the referee) although it is true in the lattice case. 
Definitions. We say that $a$ and $b$ split and write $a \leftrightarrow b$ if there exist mutually disjoint elements $a_{1}, b_{1}, c$ such that $a=a_{1}+c$ and $b=b_{1}+c$. Two derivables $x, y$ are simultaneous (denoted by $x \leftrightarrow y$ ) if $x(E) \leftrightarrow y(F)$ for every $E, F \in B(C)$. More generally $\left\{x_{\lambda}: \lambda \in \Lambda\right\}$ are simultaneous if $x_{\lambda} \leftrightarrow x_{\mu}$ for every $\lambda, \mu \in \Lambda$.

\section{Functions of simultaneous derivables.}

Definitions. Given a derivable $x$ and a complex Borel function $u$, we define the derivable $u(x)$ by $u(x)(E)=x\left[u^{-1}(E)\right]$ for all $E \in B(C)$. Let $\psi$ be a complex Borel function of $n$ complex variables. If $x$ is a derivable and $\left\{u_{i}: i=1, \cdots, n\right\}$ are complex Borel functions, then we define the derivable

$$
\psi\left(u_{1}(x), \cdots, u_{n}(x)\right)=\psi\left(u_{1}, \cdots, u_{n}\right)(x) .
$$

Lemma 3.1. Let $x$ be a derivable and let $E \in B(C)$ satisfy $x(E)=1$. If two Borel functions $f, g$ are equal on $E$, then $f(x)=g(x)$. Conversely, if $f(x)=g(x)$ then $x\{\omega: f(\omega) \neq g(\omega)\}=0$.

Proof. For $F \in B(C)$

$$
\begin{aligned}
f(x)(F) & =x\left[f^{-1}(F)\right]=x(E) \wedge x\left[f^{-1}(F)\right]=x\left[E \cap f^{-1}(F)\right]=x\left[E \cap g^{-1}(F)\right] \\
& =x(E) \wedge x\left[g^{-1}(F)\right]=g(x)(F) .
\end{aligned}
$$

Conversely, suppose $f(x)=g(x)$. Define the Borel function $f_{0}: f_{0}(\omega)=0$ for all $\omega \in C$ and apply the definition to obtain

$$
\begin{aligned}
x\{\omega: f(\omega) \neq g(\omega)\} & =x\left\{\omega:(f-g)(\omega) \in\{0\}^{\prime}\right\}=(f(x)-g(x))\left(\{0\}^{\prime}\right) \\
& =(f-f)(x)\left(\{0\}^{\prime}\right)=f_{0}(x)\left(\{0\}^{\prime}\right)=x\left(f_{0}^{-1}\{0\}^{\prime}\right)=x(\varnothing)=0 .
\end{aligned}
$$

THEOREM 3.2. If $\psi$ is a complex $n$-dimensional Borel function and

$$
\left\{x_{i}: i=1, \cdots, n\right\}
$$

are simultaneous derivables then $\psi\left(x_{1}, \cdots, x_{n}\right)$ is a well defined derivable. In fact, if $x_{i}=u_{i}(x)$ (Theorem 3.3 in $[8]\left({ }^{3}\right) \psi\left(x_{1}, \cdots, x_{n}\right)(F)=x\left\{\omega: \psi\left(u_{1}(\omega), \cdots, u_{n}(\omega)\right) \in F\right\}$ for all $F \in B(C)$.

Proof. We shall prove this theorem for $n=2$. The extension to arbitrary $n$ is obvious. By Theorem 3.2 and Proposition 3.15 in [8] there exists a smallest Boolean sub $\sigma$-algebra $A$ containing the range of both $x_{1}$ and $x_{2}$ and a derivable $y$ whose range is $A$. Applying Proposition 3.16 of [8] there exist Borel functions $v_{1}, v_{2}$ such that $x_{i}=v_{i}(y), i=1,2$, Now since $x_{i}=u_{i}(x)$, the range of each $x_{i}$ is contained in the range of $x$ and therefore $A$ is contained in the range of $x$. Again

$\left({ }^{3}\right)$ Although the theorems and propositions in [8] are proved only for observables, the generalization to derivables is easily accomplished (see [4]). We will henceforth assume this generalization has been made. 
by Proposition 3.16 of [8], there is a Borel function $u$ such that $y=u(x)$. By the definition, $u_{i}(x)=x_{i}=v_{i}(y)=v_{i}(u(x))=v_{i} \circ u(x)$. Let $E_{i}=\left\{\omega: u_{i}(\omega) \neq v_{i} \cdot u(\omega)\right\}$, $i=1,2$ and apply Lemma 3.1 to get $x\left(E_{i}\right)=0$. If $E=E_{1} \cup E_{2}$, then $x(E)=0$. Finally, for $F \in B(C)$ we have

$$
\begin{aligned}
y\left(\left\{\omega: \psi\left(v_{1}(\omega), v_{2}(\omega)\right) \in F\right\}\right) & =x\left(u^{-1}\left\{\omega: \psi\left(v_{1}(\omega), v_{2}(\omega)\right) \in F\right\}\right) \\
& =x\left[\left(v_{1} \circ u, v_{2} \circ u\right)^{-1} \psi^{-1}(F) \cap E^{\prime}\right] \\
& =x\left[\left(u_{1}, u_{2}\right)^{-1} \psi^{-1}(F)\right] \\
& =\psi\left(u_{1}(x), u_{2}(x)\right)(F)=\psi\left(x_{1}, x_{2}\right)(F) .
\end{aligned}
$$

Hence $\psi\left(x_{1}, x_{2}\right)$ is independent of the choice of $x$ and $u_{i}$ and is therefore well defined.

The following theorem is a generalization of Theorem 3.4 in [8].

THEOREM 3.3. Let $K=\left\{x_{\lambda}: \lambda \in \Lambda\right\}$ be a collection of simultaneous derivables. There exists a measurable space $(\Omega, A), a \sigma$-homomorphism $h$ of $A$ into $L$, and $A$-measurable complex valued functions $\left\{f_{\lambda}: \lambda \in \Lambda\right\}$ such that for any $n$-dimensional complex Borel function $\psi$ and any $n$ derivables $x_{1}, \cdots, x_{n} \in K$ we have $\psi\left(x_{1}, \cdots, x_{n}\right)(E)=h\left\{\omega:\left(f_{1}(\omega), \cdots, f_{n}(\omega)\right) \in \psi^{-}(E)\right\}$ for all $E \in B(C)$.

Proof. If $n=1$, the theorem follows directly from Theorem 3.4 in [8]. We shall prove the result for $n=2$, the extension to arbitrary $n$ being obvious. Let $(\Omega, A)$ be the measurable space which exists according to Theorem 3.4 in [8]. The smallest Boolean sub $\sigma$-algebra $B$ containing the range of both $x_{1}$ and $x_{2}$ is a separable subset of the smallest Boolean sub $\sigma$-algebra containing the ranges of all the $x_{\lambda}$ 's. By Proposition 3.15 [8] B is precisely the range of a derivable $z$ and by Proposition 3.16 [8] there exist Borel functions $u_{i}$ such that $x_{i}=u_{i}(z), i=1,2$. Since $z \leftrightarrow x_{\lambda}, \lambda \in \Lambda$, there exist $A$-measurable functions $f, f_{i}$ such that $z(E)$ $=h\left[f^{-1}(E)\right]$ and $x_{i}(E)=h\left[f_{i}^{-1}(E)\right]$ for all $E \in B(C)$. Therefore $h\left[f_{i}^{-1}(E)\right]=x_{i}(E)$ $=u_{i}(z)(E)=h\left[\left(u_{i} \circ f\right)^{-1}(E)\right]$ and by the uniqueness part of Proposition 3.3 [8] we have $h\left\{\omega: f_{i}(\omega) \neq u_{i} \circ f(\omega)\right\}=0$. Finally,

$$
\begin{aligned}
\psi\left(x_{1}, x_{2}\right)(E) & =z\left\{\omega: \psi\left(u_{1}(\omega), u_{2}(\omega)\right) \in E\right\} \\
& =h\left\{\omega:\left(u_{1}(f)(\omega), u_{2}(f)(\omega) \in \psi^{-1}(E)\right\}\right. \\
& =h\left\{\omega:\left(f_{1}(\omega), f_{2}(\omega)\right) \in \psi^{-1}(E)\right\} .
\end{aligned}
$$

We denote the correspondence between $x_{\lambda}$ and $f_{\lambda}$ in the above theorem by $x_{\lambda} \sim f_{\lambda}$.

We close this section with a theorem which gives the expected relationship between derivables and observables.

THEOREM 3.4. Every derivable $x$ admits a unique representation 


$$
x=x_{1}+i x_{2},
$$

where $x_{1}, x_{2}$ are observables and $x_{1} \leftrightarrow x_{2}$.

Proof. Denote the complex conjugate of a scalar $\lambda$ by $\lambda^{*}$ and let $f^{*}$ be the Borel function $f^{*}(\lambda)=\lambda^{*}$. It is easy to check that $x_{1}=\left[x+f^{*}(x)\right]$ and $x_{2}$ $=i / 2\left[f^{*}(x)-x\right]$ are simultaneous observables and, of course, $x=x_{1}+i x_{2}$. To prove uniqueness, suppose $x=y_{1}+i y_{2}$ where $y_{1} \leftrightarrow y_{2}$ are observables. Then there exists an observable $y$ and real Borel functions $u_{1}, u_{2}$ such that $y_{1}$ $=u_{1}(y), y_{2}=u_{2}(y)$. Then

$$
f^{*}(x)=f^{*} \circ\left(u_{1}+i u_{2}\right)(y)=\left(u_{1}-i u_{2}\right)(y)=y_{1}-i y_{2} .
$$

Adding $x$ to $f^{*}(x)$ we get $y_{1}=1 / 2\left[x+f^{*}(x)\right]=x_{1}$. Subtracting $f^{*}(x)$ from $x$ we get $y_{2}=i / 2\left[f^{*}(x)-x\right]=x_{2}$.

\section{The spectrum.}

Definitions. If $x$ is a derivable and $E \in B(C)$, then $E$ is $x-n u l l$ if $x(E)=0$. The union $\rho(x)$ of all open $x$-null sets of $x$ is the resolvent set of $x$. The complement $\sigma(x)$ of $\rho(x)$ is the spectrum of $x$.

It is easy to show that a derivable is an observable if and only if its spectrum is real. It may also be shown that if $L$ has an infinite number of disjoint nonzero elements, then any nonempty closed subset of the complex plane is the spectrum of some derivable $([4])$. We will henceforth assume that $L$ has at least two elements. Then since $x[\sigma(x)]=1$, we have $\sigma(x) \neq \varnothing$.

DEFINITIONS. If the closed set $\sigma(x)$ is bounded, then $x$ is a bounded derivable. If $x$ is bounded, we define the norm of $x$ to be $|x|=\sup \{|\lambda|: \lambda \in \sigma(x)\}$. If $\lambda \in C$ and $x(\lambda) \neq 0$ then $\lambda$ is in the point spectrum $\sigma_{p}(x)$ of $x$, and of course, $\sigma_{p}(x) \subset \sigma(x)$. If $\lambda \in \sigma(x)$ and $\lambda \notin \sigma_{p}(x)$ then $\lambda$ is in the continuous spectrum $\sigma_{c}(x)$ of $x$.

Suppose $\lambda$ is an isolated point of $\sigma(x)$. Then there exists an $\varepsilon>0$ such that $E-\{\lambda\} \subset \rho(x)$ where $E=\{\omega:|\omega-\lambda|<\varepsilon\}$. Now suppose $\lambda \in \sigma_{c}(x)$. Then $x(E)=x(\{\lambda\})+x(E-\{\lambda\})=0$ which is impossible. This contradiction proves that isolated points of $\sigma(x)$ are in $\sigma_{p}(x)$.

THEOREM 4.1. (i) If $K=\left\{x_{\lambda}: \lambda \in \Lambda\right\}$ is a set of simultaneous derivables, there exist A-measurable functions $\left\{f_{\lambda}: \lambda \in \Lambda\right\}$ on a measurable space $(\Omega, A)$ such that $x_{\lambda} \sim f_{\lambda}$ and $\sigma\left(x_{\lambda}\right)=\operatorname{cl} f_{\lambda}(\Omega)$. (ii) If $x \sim f$, then $\sigma(x)=\bigcap\{\operatorname{cl} f(\Gamma): h(\Gamma)=1\}$.

Proof. (i) Suppose $x \in K$ and $x \sim f_{1}$. Since $x[\rho(x)]=0$, denoting

$$
N=f_{1}^{-1}[\rho(x)]
$$

we have $h(N)=0$. Suppose $\lambda \in \sigma(x)$ and let $f(\omega)=f_{1}(\omega)$ for $\omega \in N^{\prime}$ and $f(\omega)=\lambda$ otherwise. Since we have only altered $f_{1}$ on a set $N$ for which $h(N)=0, x \sim f$. Now $f^{-1}[\rho(x)]=\varnothing$ and hence $\rho(x) \cap f(\Omega)=\varnothing$. Therefore, $\rho(x) \cap \mathrm{cl} f(\Omega)=\varnothing$ since $\rho(x)$ is open. Thus $\rho(x) \subset[\operatorname{cl} f(\Omega)]^{\prime}$ and $\operatorname{cl} f(\Omega) \subset \sigma(x)$. In the other direction, $f^{-1}\left[(\operatorname{cl} f(\Omega))^{\prime}\right]=\varnothing$. Therefore, $0=h\left\{f^{-1}\left[(\operatorname{cl} f(\Omega))^{\prime}\right]\right\}=x\left[(\operatorname{cl} f(\Omega))^{\prime}\right]$ and hence 
(cl $f(\Omega))^{\prime} \subset \rho(x)$ or $\sigma(x) \subset \mathrm{cl} f(\Omega)$. (ii) Suppose $h(\Gamma)=1$, and $F=[\mathrm{cl} f(\Gamma)]^{\prime}$. Since $f^{-1}(F) \subset \Gamma^{\prime}, 0=h\left[f^{-1}(F)\right]=x(F)$. Hence $[\mathrm{cl} f(\Gamma)]^{\prime} \subset \rho(x)$ and $\sigma(x) \subset \operatorname{cl} f(\Gamma)$. Thus $\sigma(x) \subset \bigcap\{\mathrm{cl} f(\Gamma): h(\Gamma)=1\}$. In the other direction,

$$
0=x[\rho(x)]=h\left[f^{-1}(\rho(x))\right] .
$$

Letting $\Gamma=f^{-1}(\rho(x))^{\prime}, h(\Gamma)=1$ and $\rho(x) \cap f(\Gamma)=\varnothing$. Hence $\rho(x) \cap \operatorname{cl} f(\Gamma)=\varnothing$ and cl $f(\Gamma) \subset \sigma(x)$. Therefore $\bigcap\{\mathrm{cl} f(\Gamma): h(\Gamma)=1\} \subset \sigma(x)$.

COROLlary. (i) If $x_{1} \leftrightarrow x_{2}$ are derivables, one of which is bounded, then $\sigma\left(x_{1}+x_{2}\right) \subset \sigma\left(x_{1}\right)+\sigma\left(x_{2}\right)$ and $\sigma\left(x_{1} x_{2}\right) \subset \sigma\left(x_{1}\right) \sigma\left(x_{2}\right)$. (ii) If $x_{1}$ and $x_{2}$ are both bounded, then $\left|x_{1}+x_{2}\right| \leqq\left|x_{1}\right|+\left|x_{2}\right|$ and $\left|x_{1} x_{2}\right| \leqq\left|x_{1}\right|\left|x_{2}\right|$.

Proof. By Theorem 3.3 there exist functions $g_{i}$ such that $x_{i} \sim g_{i}, i=1,2$, and $x_{1}+x_{2} \sim g_{1}+g_{2}$. As in the proof of Theorem 4.1 (i) there exist functions $f_{i}$ such that $\sigma\left(x_{i}\right)=\operatorname{cl} f_{i}(\Omega)$ and $g_{i}(\omega)=f_{i}(\omega)$ for $\omega \in N_{i}^{\prime}$ where $h\left(N_{i}\right)=0, i=1,2$. Therefore $x_{1}+x_{2} \sim f_{1}+f_{2}$. Applying Theorem 4.1 (ii) and the fact that either $\operatorname{cl} f_{1}(\Omega)$ or $\operatorname{cl} f_{2}(\Omega)$ is compact we obtain $\sigma\left(x_{1}+x_{2}\right)=\bigcap\left\{\mathrm{cl}\left(f_{1}+f_{2}\right)(\Gamma): h(\Gamma)=1\right\}$ $\subset \bigcap\left\{\operatorname{cl}\left(f_{1}(\Gamma)+f_{2}(\Gamma)\right): h(\Gamma)=1\right\}=\bigcap\left\{\operatorname{cl} f_{1}(\Gamma)+\operatorname{cl} f_{2}(\Gamma): h(\Gamma)=1\right\} \subset \operatorname{cl} f_{1}(\Omega)$ $+\operatorname{cl} f_{2}(\Omega)=\sigma\left(x_{1}\right)+\sigma\left(x_{2}\right)$. The proof of the other part is similar. (ii) follows from (i).

The remainder of this section points out the striking similarity between observables and self-adjoint operators (unbounded, in general). This similarity results from the fact that observables act very much like spectral measures (cf. [3]). Indeed if $L$ is the collection of closed subspaces of a Hilbert space, this is precisely what they are. (cf.[5] or [8].) We first prove a spectral mapping theorem.

THEOREM 4.2. Let $x$ be a derivable and $u$ a complex Borel function. (i) $\sigma(u(x)) \subset \operatorname{cl} u(C)$; (ii) if $E \in B(C)$ and $x(E)=1$, then $\sigma(u(x)) \subset \mathrm{cl} u(E)$; (iii) if $u$ is continuous, then $\sigma(u(x))=\operatorname{cl} u(\sigma(x))$.

Proof. (i) Suppose $\lambda \in \sigma(u(x))$. Then if $\lambda \in E$ and $E$ is open, we have, $0 \neq u(x)(E)=x\left[u^{-1}(E)\right]$. Therefore, $u^{-1}(E) \neq \varnothing$ and $E \cap u(C) \neq \varnothing$. Thus $\lambda \in \operatorname{cl} u(C)$ and $\sigma(u(x)) \subset \operatorname{cl} u(C)$. (ii) Let $\lambda_{0} \in u(E)$ and define $g(\lambda)=u(\lambda)$ if $\lambda \in E$ and $g(\lambda)=\lambda_{0}$ otherwise. By Lemma 3.1 and part (i) $\sigma(u(x))=\sigma(g(x)) \subset \operatorname{cl} g(C) \subset \operatorname{cl} u(E)$. (iii) Since $u(x)[\rho(u(x))]=0$, then $x\left\{u^{-1}[\rho(u(x))]\right\}=0$. Since $u$ is continuous, the open set $u^{-1}[\rho(u(x))]$ is $x$-null, and hence in $\rho(x)$. Therefore, $u^{-1}[\rho(u(x))] \cap \sigma(x)$ $=\varnothing$ and $\rho[u(x)] \cap u(\sigma(x))=\varnothing$. Hence $u(\sigma(x)) \subset \sigma(u(x))$ and $\operatorname{cl} u(\sigma(x)) \subset \sigma(u(x))$ since the latter is closed. The inclusion in the other direction follows from part (ii).

Corollary. (i) If $u$ is continuous on $\sigma(x)$, then $\sigma(u(x))=\mathrm{cl} u(\sigma(x))$. (ii) If $x$ is bounded and $u$ continuous on $\sigma(x)$, then $\sigma(u(x))=u(\sigma(x))$.

We next show that our definition of the spectrum of a derivable is the same as the conventional definition for linear operators.

Definitions. The identity derivable $I$ is the unique derivable with spectrum $\{1\}$. 
A derivable $y$ is an inverse of a derivable $x$ if $y \leftrightarrow x$ and $y x=I$. A derivable is invertible if it has a bounded inverse.

THEOREM 4.3. Let $x$ be a derivable. (i) $x$ has an inverse if and only if $0 \notin \sigma_{p}(x)$. (ii) If $x$ has an inverse it is unique(4). (iii) $x$ is invertible if and only if $0 \in \rho(x)$.

Proof. (i) Define the complex Borel function $f^{\prime}$ as $f^{\prime}(0)=0$ and $f^{\prime}(\lambda)=\lambda^{-1}$ for $\lambda \neq 0$. If $0 \notin \sigma_{p}(x), f^{\prime}(x) x(\{1\})=x\left\{\omega: f^{\prime}(\omega) \omega=1\right\}=x\left(\{0\}^{\prime}\right)=x(\{0\})^{\prime}=1$. Therefore $\sigma\left(f^{\prime}(x) x\right)=\{1\}$ and $f^{\prime}(x) x=I$. Conversely, suppose $0 \in \sigma_{p}(x)$ and $x$ has an inverse $y$. Let $x=u_{1}(z)$ and $y=u_{2}(z)$. Then

$$
\begin{aligned}
0 & =u_{1}(z) u_{2}(z)(\{0\})=z\left\{\omega: u_{1}(\omega) u_{2}(\omega)=0\right\} \\
& =z\left[\left\{\omega: u_{1}(\omega)=0\right\} \cup\left\{\omega: u_{2}(\omega)=0\right\}\right]=y(\{0\}) \vee x(\{0\}) .
\end{aligned}
$$

But this is a contradiction since $x(\{0\}) \neq 0$. (ii) If $x$ has an inverse then by (i) $0 \notin \sigma_{p}(x)$ and $f^{\prime}(x)$ is an inverse. Now suppose $y \leftrightarrow x$ and $y x=I$. Then $y \leftrightarrow f^{\prime}(x)$ and $f^{\prime}(x)=f^{\prime}(x)(x y)=I y=y$. (iii) If $x$ is invertible, then by (i) $0 \notin \sigma_{p}(x)$. Suppose $0 \in \sigma_{c}(x)$. Then $f^{\prime}(x)$ is the unique inverse of $x$. Denoting $\{\omega:|\omega| \leqq \varepsilon\}$ by $\Delta(\varepsilon)$ we have $0 \neq x\left[\Delta\left(n^{-1}\right)\right]=x\left[\Delta\left(n^{-1}\right)-\{0\}\right]=x\left[f^{\prime-1}(\Delta(n))^{\prime}\right]=f^{\prime}(x)\left[(\Delta(n))^{\prime}\right]$. Therefore $\sigma\left(f^{\prime}(x)\right)$ is not contained in $\Delta(n)$ for any $n$ and $f^{\prime}(x)$ is not bounded. This contradiction proves that $0 \notin \sigma_{c}(x)$ and hence $0 \in \rho(x)$. If $0 \in \rho(x)$, then $f^{\prime}(x)$ is the unique inverse of $x$ and $\sigma\left[f^{\prime}(x)\right]=f^{\prime}[\sigma(x)]$ is compact and hence $f^{\prime}(x)$ is bounded.

Corollary. If $x$ is a derivable, then $\rho(x)=\{\lambda: \lambda I-x$ is invertible $\}$. (Note, $x$ may be unbounded.)

Proof. Letting $g$ be the identity function and $f_{\lambda}$ the function identically equal to $\lambda$ we have

$$
\sigma(\lambda I-x)=\sigma\left[\left(f_{\lambda}-g\right)(x)\right]=\left(f_{\lambda}-g\right)(\sigma(x))=\lambda-\sigma(x) .
$$

But then $\lambda I-x$ is invertible if and only if $0 \notin \sigma(\lambda I-x)=\lambda-\sigma(x)$ or $\lambda \in \rho(x)$.

We may now prove a quite general spectral mapping theorem.

Corollary. Let $x$ be a derivable and $u$ a complex Borel function. Then $\sigma[u(x)]=\bigcap\{\operatorname{cl} u(E): x(E)=1\}$.

Proof. If $\lambda_{0} \in \rho[u(x)]$, then $\lambda_{0} I-u(x)$ is invertible and its unique bounded inverse is $f^{\prime}\left(\lambda_{0} I-u(x)\right)=f^{\prime} \circ\left(\lambda_{0}-u(\lambda)\right)(x)$. Letting $g=f^{\prime} \circ\left(\lambda_{0}-u\right)$ and $E=\{\omega:|\omega| \leqq|g(x)|\}$ we see that $x\left[g^{-1}(E)\right]=1$ and $|g(\lambda)| \leqq|g(x)|$ for $\lambda \in g^{-1}(E)$. Therefore $\lambda_{0} \notin \operatorname{cl} u\left[g^{-1}(E)\right]$ and $\bigcap\{\operatorname{cl} u(E): x(E)=1\} \subset \sigma[u(x)]$. Inclusion in the other direction follows from Theorem 4.2 (ii).

$\left({ }^{4}\right)$ Note that one can not prove this the conventional way. That is, if $y$ and $z$ are inverses, then $y=y(x z)=(y x) z=z$. This is because the associative law does not necessarily hold unless all three derivables are simultaneous. 


\section{Complete sets of bounded derivables.}

Definition. A set of simultaneous derivables $K$ is complete if $x \leftrightarrow K$ implies $x \in K$.

An easy Zorn's lemma argument shows that every nonempty set of simultaneous derivables is contained in a complete set.

LEMMA 5.1. A complete set $A$ of bounded derivables is a commutative normed algebra.

Proof. Defining the zero derivable 0 as the unique derivable with spectrum $\{0\}$, it is easily seen that $0, I \in A$ are the additive and multiplicative identities respectively, and that the additive inverse of $x$ is $(-1) x$. The rest of the proof is left to the reader.

We have shown that any derivable $x$ may be written uniquely in the form $x=x_{1}+i x_{2}$ where $x_{1} \leftrightarrow x_{2}$ are observables. Let us now define a new derivable $x^{*}=x_{1}-i x_{2}$ which we call the adjoint of $x$. From Theorem 3.4 we see that $x^{*}=f^{*}(x)$. By adding and subtracting $x$ and $x^{*}$ we see that $x_{1}=1 / 2\left(x+x^{*}\right)$ and $x_{2}=i / 2\left(x^{*}-x\right)$. We next show that the mapping $x \rightarrow x^{*}$ is an involution.

LEMMA 5.2. The mapping $x \rightarrow x^{*}$ is a one-one map of a complete set of derivables $A$ onto itself satisfying:
(i) $\left(x^{*}\right)^{*}=x$;
(ii) $(x+y)^{*}=x^{*}+y^{*}$;
(iii) $(\lambda x)^{*}=\lambda^{*} x^{*}$;
(iv) $(x y)^{*}=x^{*} y^{*}$;
(v) $\sigma\left(x^{*}\right)=\sigma(x)^{*}$;
(vi) $\left|x^{*} x\right|=|x|^{2}$.

Proof. If $x \in A$ then $x^{*} \in A$ since $x^{*}=f^{*}(x)$. That the mapping is one-one, onto will follow from (i).

(i) $\left(x^{*}\right)^{*}=f^{*} \circ f^{*}(x)=x$.

Statements (ii), (iii), and (iv) result from the following properties of $f^{*}$ : if $x \leftrightarrow y$ then

$$
f^{*}(x+y)=f^{*}(x)+f^{*}(y) ; f^{*}(x y)=f^{*}(x) f^{*}(y) ; f^{*}(\lambda x)=\lambda^{*} x^{*} .
$$

(v) Since $f^{*}$ is a continuous function, the spectral mapping theorem gives:

$$
\sigma\left(x^{*}\right)=\sigma\left(f^{*}(x)\right)=\operatorname{cl} f^{*}(\sigma(x))=\sigma(x)^{*} .
$$

(vi) $\left|x^{*} x\right| \leqq\left|x^{*}\right||x|=|x|^{2}$. Now suppose $E$ is open and $x(E) \neq 0$. Then $E E^{*}$ is open and $0 \neq x(E) \leqq x\left\{\omega: \omega \omega^{*} \in E E^{*}\right\}=x x^{*}\left(E E^{*}\right)$. Hence $\lambda \in \sigma(x)$ implies $|\lambda|^{2} \in \sigma\left(x x^{*}\right)$ and $|\sigma(x)|^{2} \subset \sigma\left(x x^{*}\right)$. Therefore, $|x|^{2} \leqq\left|x^{*} x\right|$.

Definition. If $f_{n}, f$ are Borel functions we say that $f_{n} \rightarrow f$ with respect to a derivable $x$ if for every $\varepsilon>0$ there exists a positive integer $n(\varepsilon)$ such that

for all $n \geqq n(\varepsilon)$.

$$
x\left\{\omega:\left|f_{n}(\omega)-f(\omega)\right|>\varepsilon\right\}=0
$$

LEMMA 5.3. If $f_{n}, f$ are Borel functions, then $f_{n} \rightarrow f$ with respect to $x$ if and only if $f_{n}(x) \rightarrow f(x)$ in norm. 
Proof. Suppose $f_{n} \rightarrow f$ with respect to $x$. Let $\varepsilon, n(\varepsilon)$ be as in the definition and let $\Delta(\varepsilon)=\{\lambda:|\lambda| \leqq \varepsilon\}$. Then

$$
\begin{aligned}
{\left[f_{n}(x)-f(x)\right]\left(\Delta(\varepsilon)^{\prime}\right) } & =x\left\{\omega: f_{n}(\omega)-f(\omega) \in \Delta(\varepsilon)^{\prime}\right\} \\
& =x\left\{\omega:\left|f_{n}(\omega)-f(\omega)\right|>\varepsilon\right\}=0
\end{aligned}
$$

for all $n \geqq n(\varepsilon)$. Therefore, for $n \geqq n(\varepsilon), \sigma\left[f_{n}(x)-f(x)\right] \subseteq \Delta(\varepsilon)$, and $\left|f_{n}(x)-f(x)\right|<\varepsilon$, i.e., $f_{n}(x) \rightarrow f(x)$. The sufficiency is proved by reversing the above steps.

THEOREM 5.4. If $x_{i}$ is a sequence of bounded simultaneous derivables converging to the bounded derivable $x$ and $y \leftrightarrow x_{i}$, then $y \leftrightarrow x$.

Proof. There exists a derivable $z$ and complex Borel functions $u, u_{n}$, such that $x_{n}=u_{n}(z)$ and $y=u(z)$. Since $\left(x_{n}\right)$ is Cauchy, there exist positive integers $n(p)$, $p=1,2, \cdots$, such that $n, m \geqq n(p)$ implies $\left|u_{n}(z)-u_{m}(z)\right| \leqq 1 / p$. Therefore, in the notation of the previous lemma we have $\sigma\left[u_{n}(z)-u_{m}(z)\right] \subset \Delta\left(p^{-1}\right)$ and

$$
0=\left[u_{n}(z)-u_{m}(z)\right]\left(\Delta\left(p^{-1}\right)^{\prime}\right)=z\left\{\omega:\left|u_{n}(\omega)-u_{m}(\omega)\right|>p^{-1}\right\} .
$$

Denoting $\left\{\omega:\left|u_{n}(\omega)-u_{m}(\omega)\right|>p^{-1}\right\}=N(p)$ we have $\left|u_{n}(\omega)-u_{m}(\omega)\right| \leqq p^{-1}$ on $C-N(p)$ and $z[N(p)]=0$. Now if $N=\bigcup N(p)$, then $z(N)=\vee z[N(p)]=0$. We assert that $\left(u_{n}\right)$ is uniformly Cauchy on $C-N$. Indeed, let $\varepsilon>0$ be given. Then there is an integer $q$ such that $q^{-1}<\varepsilon$ and if $n, m>n(q)$, we have $\left|u_{n}(\omega)-u_{m}(\omega)\right| \leqq q^{-1}<\varepsilon$ on $C-N(q)$ and hence on $C-N$. Therefore, $u_{n}(\omega)$ converges uniformly to a measurable function $v(\omega)$ on $C-N$. Hence $u_{n} \rightarrow v$ with respect to $z$ and by Lemma $5.3, u_{n}(z) \rightarrow v(z)$. But $u_{n}(z)=x_{n} \rightarrow x$. Therefore, $x=v(z)\left({ }^{5}\right)$ and since $y=u(z), x \leftrightarrow y$.

Corollary. A complete set of bounded derivables $A$ is metrically complete.

Proof. Let $\left(x_{n}\right)$ be Cauchy in $A$ and let $x_{n}=u_{n}(x)$. As in the proof of Theorem 5.4, $\left(u_{n}(\omega)\right)$ is uniformly Cauchy on $C-N$ where $x(N)=0$. Therefore there exists a Borel function $u$ such that $u_{n}(\omega) \rightarrow u(\omega)$ uniformly on $C-N$ and hence with respect to $x$. By Lemma 5.3, $u_{n}(x) \rightarrow u(x)$. Now suppose $z \in A$, then $z \leftrightarrow x_{n}$. By Lemma 5.4, $u(x) \leftrightarrow z$. Therefore $u(x) \in A$ and $A$ is metrically complete.

We now prove a representation theorem for complete sets of bounded derivables.

THEOREM 5.5. Every complete set of bounded derivables $A$ is isometrically *-isomorphic with the continuous complex-valued functions on a compact Hausdorff space. Under this isomorphism the observables correspond to continuous real-valued functions.

(5) Added in proof. The author recently discovered that to justify this step he implicitly assumed the following quite mild axiom: if a sequence of bounded simultaneous derivables converges to two derivables $x$ and $y$, then $x=y$. This axiom is needed only at this point, and the results of the following sections do not depend upon it. 
Proof. By Lemmas 5.1, 5.2 and the previous corollary, $A$ is a commutative $B^{*}$-algebra and the theorem follows from the Gelfand-Naimark theorem (cf. $[3$, II, Theorem IX, 3.7]).

\section{The states.}

Definitions. The distribution of a derivable $x$ in the state $m$ (or under $m$ ) is the probability measure $m_{x}(\cdot)=m[x(\cdot)]$. If $u$ is a complex Borel function, the expectation or average value of $u(x)$ under $m$, if it exists, is

$$
m[u(x)]=\int_{c} u(\lambda) m_{x}(d \lambda) .
$$

When we write $m(x)$ we are assuming that the expectation exists and is finite under $m$ and we shall use the notation $M_{x}=\{m \in M: m(x)$ exists, finite $\}$. If $x$ is bounded then, of course, $M_{x}=M$.

THEOREM 6.1. Let $A$ be a complete set of bounded derivables. Then the mapping $m: A \rightarrow C$ defined by $x \rightarrow m(x)$ is a bounded linear functional on $A$ which satisfies $|x|=\sup \{|m(x)|: m \in M\}$.

Proof. Suppose $x, y \in A$ and $x=u_{1}(z), y=u_{2}(z)$. We prove linearity as follows:

$$
\begin{aligned}
m(x+y) & =m\left[u_{1}(z)+u_{2}(z)\right]=\int\left[u_{1}(\lambda)+u_{2}(\lambda)\right] m_{z}(d \lambda) \\
& =\int u_{1}(\lambda) m_{z}(d \lambda)+\int u_{2}(\lambda) m_{z}(d \lambda)=m\left[u_{1}(z)\right]+m\left[u_{2}(z)\right] \\
& =m(x)+m(y) ; \\
m(\alpha x) & =\int \alpha \lambda m_{x}(d \lambda)=\alpha m(x) . \mathbf{1}
\end{aligned}
$$

The following proves boundedness:

$$
|m(x)|=\left|\int_{\sigma(x)} \lambda m_{x}(d \lambda)\right| \leqq \int_{\sigma(x)}|\lambda| m_{x}(d \lambda) \leqq|x| .
$$

Hence $\sup \{|m(x)|: m \in M\} \leqq|x|$. Let $\varepsilon>0$ be given, let $\lambda_{0} \in \sigma(x)$, and define $E=\left\{\omega:\left|\omega-\lambda_{0}\right|<\varepsilon\right\}$. Since $E$ is open $x(E) \neq 0$ and hence there exists $m \in M$ such that $m[x(E)]=1$. Therefore, $|m(x)|=\left|\int_{E} \lambda m_{x}(d \lambda)\right| \geqq\left|\lambda_{0}\right|-\varepsilon$ and $\left|\lambda_{0}\right| \leqq|m(x)|+\varepsilon \leqq \sup \{|m(x)|: m \in M\}+\varepsilon$. Thus

$$
\sup \{|\lambda|: \lambda \in \sigma(x)\} \leqq \sup \{|m(x)|: m \in M\}+\varepsilon
$$

and since $\varepsilon>0$ was arbitrary, $|x| \leqq \sup \{|m(x)|: m \in M\}$.

It is routine to check that $M$ is a strongly convex set, i.e., if $\left(m_{i}\right)$ is a sequence of states and $\left(\lambda_{i}\right)$ a sequence of positive numbers whose sum is one, then the mapping $a \rightarrow \sum \lambda_{i} m_{i}(a)$ is a state. 
Definition. If $x$ is a derivable, we define $V(x)=\operatorname{cl}\left\{m(x): m \in M_{x}\right\}$.

THEOREM 6.2. The set $V(x)$ is the closed convex hull of $\sigma(x)$. (Note that $x$ may be unbounded.)

Proof. We first prove that if a derivable $y$ is not invertible, then given $\varepsilon>0$ there exists an $m \in M$ such that $|m(y)| \leqq \varepsilon$. Indeed, suppose $y$ is such a derivable, $\varepsilon>0$ is given, and $E=\{\lambda:|\lambda|<\varepsilon\}$. Then $0 \in \sigma(y)$ by Theorem 4.3. Since $E$ is open $y(E) \neq 0$ and there exists an $m \in M$ such that $m[y(E)]=1$. Then $|m(y)|$ $=\left|\int_{E} \lambda m_{y}(d \lambda)\right| \leqq \varepsilon$. We now show that $\sigma(x) \subset V(x)$. Suppose $\lambda \notin V(x)$, then $\operatorname{dist}(\lambda, V(x))=d>0$. Therefore $|m(x)-\lambda| \geqq d$ or $|m(x-\lambda I)|=|m(x)-\lambda m(I)|$ $\geqq d$ for every $m \in M$ for which $m(x)$ exists. By the first part of this proof, $x-\lambda I$ is invertible and therefore $\lambda \in \rho(x)$. Thus $V(x)^{\prime} \subseteq \rho(x)$ and $\sigma(x) \subseteq V(x)$. We next show that $\{m(x): m \in M\}$ is convex. Let $\lambda_{1}=m_{1}(x)$ and $\lambda_{2}=m_{2}(x)$ where $m_{1}$, $m_{2} \in M$. Given $0 \leqq \alpha \leqq 1$, define $m_{3}(a)=\alpha m_{1}(a)+(1-\alpha) m_{2}(a)$ for all $a \in L$. Now $m_{3} \in M$ and

$$
m_{3}(x)=\alpha m_{1}(x)+(1-\alpha) m_{2}(x)=\alpha \lambda_{1}+(1-\alpha) \lambda_{2} \in\{m(x): m \in M\}
$$

and thus $\{m(x): m \in M\}$ is convex. Since the closure of a convex set is convex, we see that $V(x)$ is a closed convex set containing $\sigma(x)$. Therefore $V(x)$ contains the closed convex hull $A$ of $\sigma(x)$. Suppose $\mu \in V(x)$ but $\mu \notin A$. Then $\mu$ and $A$ may be strictly separated by a closed hyperplane (straight line). If $m \in M$ and $m(x)$ exists, we have

$$
m(x)=\int_{\sigma(x)} \lambda m_{x}(d \lambda)=\int_{H} \lambda m_{x}(d \lambda),
$$

where $H$ denotes the closed half-plane, determined by the hyperplane, which contains $A$. We thus have $m(x) \in H$, for all $m \in M$ for which $m(x)$ exists. Therefore $\mu \in H$ which is a contradiction. Hence $V(x) \subset A$.

COROLlaRY. If $x$ is bounded, the extreme points of $V(x)$ are contained in $\sigma(x)$.

Proof. This follows from a standard corollary to the Krein-Milman theorem.

One might think that it is possible to have an unbounded derivable $x$ for which $m(x)$ exists for every $m \in M$. We now prove that this is impossible.

THEOREM 6.3. A derivable $x$ is bounded if and only if $m(x)$ exists for every $m \in M$.

Proof. The necessity is trivial; to prove the sufficiency, suppose $x$ is unbounded. Then there exist distinct numbers $\lambda_{n} \in \sigma(x)$ such that $\left|\lambda_{n}\right|>2^{n+1}, n=1,2, \cdots$. Let $E_{n}$ be disjoint open disks of diameter less than one centered at $\lambda_{n}$ and let $a_{n}=x\left(E_{n}\right) \neq 0$. Now there exist $m_{j} \in M$ such that $m_{j}\left(a_{j}\right)=1$. Since $M$ is strongly convex $m=\Sigma_{1}^{\infty} 2^{-j} m_{j}$ is in $M$. Since $a_{i} \perp a_{j}, m_{i}\left(a_{j}\right)=0$ for $i \neq j$ and $m\left(a_{j}\right)=2^{-j}$. Now suppose $m(x)$ exists and therefore, $\int|\lambda| m_{x}(d \lambda)$ exists. But 


$$
\begin{aligned}
\int|\lambda| m_{x}(d \lambda) & \geqq \sum_{i=1}^{\infty} \int_{E_{i}}|\lambda| m_{x}(d \lambda) \\
& \geqq \sum_{i=1}^{\infty}\left(2^{i+1}-1\right) 2^{-i}=+\infty
\end{aligned}
$$

This contradiction shows that $m(x)$ does not exist and finishes the proof.

Corollary. Let $X_{i}, i=1, \cdots, n$, be a finite collection of complete sets of derivables and $Y \subset \bigcup X_{i}$. If $\sup \{|m(x)|: x \in Y\}<\infty$ for all $m \in M$, then

$$
\sup \{|x|: x \in Y\}<\infty \text {. }
$$

Proof. Since $m(x)$ exists for every $m \in M$, by Theorem 6.3 every $x \in Y$ is bounded. Hence $Y \cap X_{i}, i=1, \cdots, n$, is contained in a complete set of bounded derivables $\bar{X}_{i}$. But $\bar{X}_{i}$ is a Banach space and $M$ is a norm determining subset of the dual of $X_{i}$. By the uniform boundedness theorem (cf. $[7,4.4-B]$ )

$$
\sup \left\{|x|: x \in Y \cap X_{i}\right\}<\infty
$$

and hence $\sup \{|x|: x \in Y\}<\infty$.

\section{The weak topology.}

DEFINITION. A net $m_{\alpha}$ of states converges weakly to the state $m$ if $m_{\alpha}(a) \rightarrow m(a)$ for every $a \in L$. The weak topology of $M$ is the unique topology for which nets converge weakly.

It is easy to see that a neighborhood basis for the weak topology is given by sets of the form

$$
N\left(m_{0} ; a_{1}, \cdots, a_{j}, \varepsilon\right)=\left\{m \in M:\left|m\left(a_{i}\right)-m_{0}\left(a_{i}\right)\right|<\varepsilon, \quad i=1, \cdots, j\right\} .
$$

The weak topology is Hausdorff, for if $m_{1} \neq m_{2}$ there exists an $a \in L$ such that $m_{1}(a) \neq m_{2}(a)$. Letting $\varepsilon=1 / 2\left|m_{1}(a)-m_{2}(a)\right|$ we have $N\left(m_{1}: a, \varepsilon\right) \cap N\left(m_{2} ; a, \varepsilon\right)$ $=\varnothing$.

A simple function is a finite linear combination of indicator functions. The following lemma will prove useful.

LEMMA 7.1. If $x$ is a bounded derivable, there exists a sequence of simple functions of $x$ converging to $x$ in norm.

Proof. Let $g(\lambda)=\lambda$ for $|\lambda| \leqq|x|$, and $g(\lambda)=0$ for $|\lambda|>|x|$. Then by Lemma $3.1 g(x)=x$. Since $g$ is a bounded Borel function, there exists a sequence of simple functions $s_{n}$ which converge uniformly to $g$. By Lemma 5.3, $s_{n}(x) \rightarrow g(x)$ $=x$.

Notation. We denote by $x_{a}$ the derivable which satisfies $x_{a}(\{1\})=a$ and $x_{a}(\{0\})=a^{\prime}$.

THEOREM 7.2. The net $m_{\alpha} \rightarrow m$ weakly if and only if $m_{\alpha}(x) \rightarrow m(x)$ for every bounded derivable $x$. 
Proof. To prove sufficiency we have $m_{a}(a)=m_{a}\left(x_{a}\right) \rightarrow m\left(x_{a}\right)=m(a)$. Conversely, if $x$ is bounded, applying Lemma 7.1 there exists a sequence of simple functions $s_{j}$ of $x$ such that $s_{j} \rightarrow x$. Given $\varepsilon>0$, let $n$ be so large that $\left|x-s_{n}\right|<\varepsilon / 3$. Suppose $s_{n}=\sum_{1}^{k} c_{i} I_{E_{i}}, c_{i} \neq 0$. Since $m_{\alpha} \rightarrow m$ weakly, there exists an $\alpha_{0}$ such that $\alpha \geqq \alpha_{0}$ implies

$$
\left|m_{\alpha}\left[x\left(E_{i}\right)\right]-m\left[x\left(E_{i}\right)\right]\right|<\varepsilon /\left(3 k\left|c_{i}\right|\right), \quad i=1, \cdots, k .
$$

Hence, if $\alpha \geqq \alpha_{0}$, then

$$
\begin{aligned}
\left|m_{\alpha}(x)-m(x)\right| & \leqq\left|m_{\alpha}\left(x-s_{n}\right)\right|+\left|m\left(s_{n}-x\right)\right|+\mid \sum_{c_{i}} m_{\alpha}\left[x\left(E_{i}\right)\right]-\sum c_{i} m\left[x\left(E_{i}\right)\right] \\
& \leqq\left|x-s_{n}\right|+\left|s_{n}-x\right|+\Sigma\left|c_{i}\right|\left|m_{\alpha}\left[x\left(E_{i}\right)\right]-m\left[x\left(E_{i}\right)\right]\right|<\varepsilon .
\end{aligned}
$$

Let us now view $M$ as a subset of all the bounded linear functionals $X^{\prime}$ on the collection of bounded derivables $X$. A functional $f$ on $X$ is said to be linear if $x \leftrightarrow y$ implies $f(\alpha x+\beta y)=\alpha f(x)+\beta f(y) . f$ is bounded if $|f|=\sup \{|f(x)|:|x| \leqq 1\}<\infty$. It is straightforward to show that $|f|=\inf \{K:|f(x)| \leqq K|x|, x \in X\}$, in particular, $|f(x)| \leqq|f||x|$. Although $X$ has a norm (in a sense), it is not a linear space since addition is not defined for all elements of $X$. Therefore, some care must be exercised in developing the theory. We define $(\alpha f)(x)=\alpha f(x)$ and $(f+g)(x)=f(x)+g(x)$. These are both bounded linear functional if $f, g$ are and $|\alpha f|=|\alpha||f|,|f+g| \leqq|f|+|g|$. The zero functional is defined $0(x)=0$ for all $x \in X$ and $|0|=0$. If $|f|=0$ then $f(x)=0$ for all $x \in X$ and thus $f=0$. Therefore, the bounded linear functionals $X^{\prime}$ on $X$ form a normed linear space. The proof that $X^{\prime}$ is complete in the norm topology is almost identical to the proof when $X$ is a normed linear space.

THEOREM 7.3. The normed linear space $X^{\prime}$ is complete.

Proof. Cf. [7, Theorem 4.1-A].

THEOREM 7.4. $M$ is a norm closed strongly convex subset of the surface of the unit ball $U^{\prime}$ of $X^{\prime}$.

Proof. It is routine to show that $M$ is a strongly convex subset of $U^{\prime}$. To show that $M$ is normed closed, let $\left(m_{n}\right)$ be a sequence in $M$ with $m_{n} \rightarrow f \in X^{\prime}$. For $a \in L$ define $m(a)=f\left(x_{a}\right)$. Now, $m(0)=f\left(x_{0}\right)=f(0)=0 ; m(1)=f\left(x_{1}\right)=\lim m_{n}(1)=1$. Since $0 \leqq m_{n}\left(x_{a}\right) \leqq 1$, we have $0 \leqq f\left(x_{a}\right) \leqq 1$ and $0 \leqq m(a) \leqq 1$. Letting $b=\sum a_{i}$ we have

Now,

$$
m(b)=f\left(x_{b}\right)=\lim m_{n}\left(x_{b}\right)=\lim m_{n}(b)=\lim _{n \rightarrow \infty} \sum m_{n}\left(a_{i}\right) .
$$

$$
\begin{aligned}
\left|\sum_{1}^{p} m_{n}\left(x_{a_{i}}\right)-\sum_{1}^{p} f\left(x_{a_{i}}\right)\right| & \leqq\left|m_{n}-f\right|\left|\sum_{1}^{p} x_{a_{i}}\right| \\
& =\left|m_{n}-f\right|\left|x_{b}\right|=\left|m_{n}-f\right| .
\end{aligned}
$$


Letting $p \rightarrow \infty$ we have

$$
\left|\sum_{1}^{\infty} m_{n}\left(x_{a_{i}}\right)-\sum_{1}^{\infty} f\left(x_{a_{i}}\right)\right| \leqq\left|m_{n}-f\right| \rightarrow 0
$$

as $n \rightarrow \infty$. Therefore, $m\left(\sum a_{i}\right)=\sum_{1}^{\infty} f\left(x_{a_{i}}\right)=\sum_{1}^{\infty} m\left(a_{i}\right)$. Hence $m \in M$ and $m\left(x_{a}\right)=m(a)=f\left(x_{a}\right)$. Now if $x \in X$ there exists a sequence of simple functions $s_{j}$ of $x$ which converge to $x$ by Lemma 7.1. Given $\varepsilon>0$, let $n$ be so large that $\left|s_{n}-x\right|<(\varepsilon / 2) \min \left(1,|f|^{-1}\right)$. Then

$$
\begin{aligned}
|m(x)-f(x)| & \leqq\left|m(x)-m\left(s_{n}\right)\right|+\left|m\left(s_{n}\right)-f\left(s_{n}\right)\right|+\left|f\left(s_{n}\right)-f(x)\right| \\
& \leqq\left|x-s_{n}\right|+|f|\left|s_{n}-x\right|<\varepsilon .
\end{aligned}
$$

Therefore, $m(x)=f(x)$ for all $x \in X$ and $f \in M$.

We now endow $X^{\prime}$ with a weak topology $\tau$. A neighborhood basis for $\tau$ of the origin will be sets of the form: $N\left(\varepsilon, x_{1}, \cdots, x_{n}\right)=\left\{f \in X^{\prime}:\left|f\left(x_{i}\right)\right|<\varepsilon, i=1, \cdots, n\right\}$, where $\varepsilon>0$ and $\left\{x_{i}\right\}$ is any finite subset of $X$. Now $\tau$ makes $X^{\prime}$ a locally convex space. By Theorem 7.2 we see that $\tau$ induces on $M$ a topology which is equivalent to the weak topology already defined there. We would now like to prove Alaoglu's theorem for $U^{\prime}$. Although Alaoglu's theorem as it stands is not applicable in our case since $X$ is not even a vector space, the proof holds and is identical to the standard proof.

TheOREM 7.5 (Alaoglu). $U^{\prime}$ is compact Hausdorff in the $\tau$ topology.

Proof. Cf. [3, Theorem V. 4.2.]

THEOREM 7.6. If $X^{\prime}$ with the $\tau$ topology is metrizable, then $M$ is a $\tau$-closed subset of $X^{\prime}$.

Proof. Let $\left(m_{i}\right)$ be a sequence in $M$ and suppose $m_{i} \rightarrow f \in X^{\prime}$ in the $\tau$ topology. Define $m: L \rightarrow C$ by $m(a)=f\left(x_{a}\right)$. It is easily seen that $m(0)=0, m(1)=1$, and $0 \leqq m(a) \leqq 1, a \in L$. We now show that $m$ is "countably additive." Let $\left(a_{i}\right)$ be a disjoint sequence in $L$, and $A$ the smallest Boolean $\sigma$-algebra containing $\bigcup a_{i}$. By the Loomis representation theorem, there exists a measurable space $(\Omega, S)$ and a $\sigma$-homomorphism $h$ from $S$ onto $A$. Suppose $h\left(\Lambda_{i}\right)=a_{i}$. Now $m_{i}$ induces a measure $\bar{m}_{i}$ on $S$ defined by: $\bar{m}_{i}(\Lambda)=m_{i}(h(\Lambda)), \Lambda \in S$. Defining $\bar{m}(\Lambda)=m(h(\Lambda))$ we have

$$
\lim \bar{m}_{i}(\Lambda)=\lim m_{i}(h(\Lambda))=m(h(\Lambda))=\bar{m}(\Lambda),
$$

$\Lambda \in S$. We thus have a sequence $\left(\bar{m}_{i}\right)$ of measures in $(\Omega, S)$ converging to the set function $\bar{m}$. By a theorem due to Nikodym ([3, III. 7.4]), $\bar{m}$ is countably additive on $S$. Now let us define, $\Gamma_{1}=\Lambda_{1}, \Gamma_{2}=\Lambda_{2}-\Lambda_{1}, \quad \Gamma_{3}=\Lambda_{3}-\left(\Lambda_{1} \cup \Lambda_{2}\right), \cdots$. Then $\Gamma_{1} \cap \Gamma_{j}=\varnothing i \neq j$ and $h\left(\Gamma_{i}\right)=a_{i}$. We thus have

$$
\begin{aligned}
m\left(\sum a_{i}\right) & =m\left[\sum h\left(\Gamma_{i}\right)\right]=m\left[h\left(\bigcup \Gamma_{i}\right)\right]=\bar{m}\left(\bigcup \Gamma_{i}\right) \\
& =\sum \bar{m}\left(\Gamma_{i}\right)=\sum m\left[h\left(\Gamma_{i}\right)\right]=\sum m\left(a_{i}\right) .
\end{aligned}
$$


Hence $m \in M$. Since $f\left(x_{a}\right)=m(a)=m\left(x_{a}\right)$ for every $a \in L$, by the proof of Theorem 7.4, $f(x)=m(x) x \in X$ and $f \in X^{\prime}$.

Since $M$ is a closed subset of the compact set $U^{\prime}$, we see that $M$ is a compact convex subset of $X^{\prime}$ in the weak topology. Now by the Krein-Milman theorem, $M$ is the $\tau$ closed convex hull of its extreme points.

Definition. The extreme points of $M$ are called pure states.

COROLlaRY. If $X^{\prime}$ is metrizable, then $M$ is the weakly closed convex hull of its pure states.

Now $M$ is not, in general, a closed subset of $X^{\prime}$ for the weak topology. This may be shown by considering a measurable space $(\Omega, A)$ on which there is a finitely additive measure $\mu$ which is not countably additive. $(\Omega=R$ and $A=B(R)$ would do.) One may then construct a net $m_{\alpha} \in M$ which converges weakly to a functional not in $M$. (For details, see [4].)

\section{REFERENCES}

1. G. Bodiou, Théorie dialectique des probabilités, Gauthier-Villars, Paris, 1964.

2. - Probabilité sur une treillis non modulair, Publ. Inst. Statist. Univ. Paris 6 (1957), 11-25.

3. N. Dunford and J. T. Schwartz, Linear operators. Parts I, II, Interscience, New York, $1958,1963$.

4. S. P. Gudder, A generalized probability model for quantum mechanics, Ph.D. Thesis, University of Illinois, Urbana, Ill., 1964.

5. G. W. Mackey, The mathematical foundatious of quantum mechanics, Benjamin, New York, 1963.

6. J. C. T. Pool, Simultaneous observability and the logic of quantum mechanics, Ph.D. Thesis, State University of Iowa, Iowa City, Iowa, 1963.

7. A. E. Taylor, Introduction to functional analysis, Wiley, New York, 1961.

8. V. S. Varadarajan, Probability in physics and a theorem on simultaneous observability, Comm. Pure Appl. Math. 15 (1962), 189-217.

\section{UNIVERSITY OF WISCONSIN,}

Madison, WisCONSIN 